\title{
Urinary tract infections due to coagulase-negative staphylococci
}

\author{
R. G. MITCHELL \\ From the Department of Pathology, Royal Infirmary, Bristol
}

SYNOPSIS A survey of Staphylococcus albus urinary infections is reported from a general hospital. The infection followed urethral instrumentation in $75 \%$ of the patients, and was usually caused by organisms already present in the urethra. Novobiocin-resistant strains caused infections in four out-patients with no predisposing lesions or instrumentation of the urinary tract.

Although usually regarded as harmless commensals, coagulase-negative staphylococci (Staphylococcus albus) sometimes cause infections of the urinary tract. The most likely source of the organism would seem to be the patient's own urethra, of which it is a normal inhabitant (Shackman and Messent, 1954). The alternative possibility of infection by a common exogenous strain was suggested by the work of Pereira (1962), who described cases of urinary tract infection caused by strains of Staph. albus resistant to novobiocin, sensitive to other common antibacterial agents, and serologically homogenous.

These findings prompted us to investigate Staph. albus urinary infections recognized from January 1962 to March 1963 in a laboratory serving 600 beds in a general hospital.

\section{METHODS}

Infection by Staph. albus was diagnosed when the centrifuged deposit of a freshly-collected midstream or catheter specimen of urine (usually examined within two hours) contained excess leucocytes (more than 10 per high-power field), together with numerous clusters of Gram-positive cocci, and culture yielded a profuse growth of Staph. albus without other organisms. Antibiotic resistance patterns were determined by the agar plate diffusion method, using Mast discs, including a $5 \mu \mathrm{g}$. novobiocin disc. When on occasion a culture yielded mixed strains of Staph. albus the predominant strain only was investigated.

\section{RESULTS}

BACTERIOLOGY Sixty-three urinary infections due to Staph. albus were diagnosed in 60 patients. Strains were available for study from the most recent 36 of these.

Received for publication 16 May 1963.
The majority of strains isolated showed the typical colonial and microscopic appearances of staphylococci, were catalase-positive, and gave negative tests for tube-coagulase, alpha-toxin, mannite fermentation, pigmentation on milk agar medium, and phage typability. Three strains fermented mannite. One of these was pigmented, could be phage-typed, and was probably a coagulase-negative variant of Staph. aureus. Widely differing antibiotic resistance patterns were encountered. Of 58 strains tested, 13 were sensitive to all the commonly used antibacterial drugs; 14 were resistant to one drug only (penicillin eight, streptomycin one, tetracycline one, novobiocin four); and 31 were resistant to penicillin and at least one other drug. Seventeen strains were resistant to penicillin, streptomycin, and tetracycline.

The urine white cell count varied from 10 to 20 cells/high-power field (H.P.F.) in 19 cases, 20 to 50 cells/H.P.F. in 18, 50 to 100 cells/H.P.F. in six, 100 to 200 cells/H.P.F. in 12 , and was over 200 cells/ H.P.F. in eight.

CLINICAL DETAILS The main clinical categories are shown in Table I. In $75 \%$ of the patients, infection had followed instrumentation of the urinary tract, while in all but four of the other patients there were factors which predisposed them to infections.

Forty-three $(70 \%)$ of the patients were males.

Urinary symptoms were usually slight and not always well documented, and in any case are difficult to assess after various forms of instrumentation. At least 10 patients, however, had moderately severe dysuria, with frank pyuria, and responded promptly to appropriate antibacterial therapy. In one patient visual proof of generalized cystitis was obtained at cystoscopy. 
TABLE I

MAIN CLINICAL CATEGORIES IN PRESENT SERIES

\begin{tabular}{l} 
Clinical Categories \\
\hline Previous instrumentation of urinary tract \\
Prostatectomy or resection of bladder tumour \\
Continuous urethral catheterization \\
Cystoscopy \\
Passage of sounds \\
Vaginal hysterectomy, catheterized \\
Post-partum, catheterized \\
Pyelolithotomy \\
No previous instrumentation \\
Calculus of urinary tract \\
Bladder tumour \\
Urethral stricture \\
Chronic pyelonephritis \\
Diabetes mellitus \\
Pregnancy \\
Post-partum \\
No cause found
\end{tabular}

No. of Cases

DISCUSSION

The majority of the patients were undergoing urological treatment or catheterization, with resulting trauma to the urethra which evidently was sufficient to enable commensal organisms to assume a pathogenic role. The causative strains possessed widely differing sensitivity patterns. In only a few instances could the sensitivity have been modified, or the organisms selected, by previous antibacterial therapy. Post-operative cross-infection by endemic 'hospital' strains of Staph. albus may sometimes have occurred, but there was no evidence of this. Thus, three urinary infections, which occurred simultaneously in patients in the same ward after prostatectomy, were caused by strains of Staph. albus with different sensitivity patterns. It was concluded that the infections were mostly caused by organisms already present in the urethra. This belief was supported by analysis of the previous incidence and causation of post-operative urinary infection in this hospital. During the period
1955 to 1959 , measures were introduced to preveñt cross infection, and the incidence of infection not die to Staph. albus fell from $74 \%$ to $11 \%$ (Gillespie, Linton, Miller, and Slade, 1960). In contrasi, throughout this period, Staph. albus in pure cultuse continued to infect about $10 \%$ of all prostatector patients, though mixed infections of Staph. alb with other organisms, previously common, virtualty disappeared.

Pereira (1962) isolated a novobiocin-resistaht strain of Staph. albus from 40 patients, mainty females, in a Portuguese hospital practice, accoup ing for some $4 \%$ of all urinary infections; but he not state whether other strains of Staph. albus caused infection. His strains may have been 40 usually invasive and capable of giving rise to cross-infection, but he gave no clinical or epidemierlogical details on which an opinion can be based. 9

In the present series there were four infections by novobiocin-resistant strains. All were in out-patier (three females and one male) and in none was predisposing cause found for the infection. Symptoms were sufficiently severe to require antibacteriol treatment in three of these patients. These limited findings are in line with Pereira's observations.

I wish to thank Professor W. A. Gillespie, in whळ్ department this work was carried out, and Dr. K. Linton for their invaluable advice and helpful criticisn.

\section{REFERENCES}

Gillespie, W. A., Linton, K. B., Miller, A., and Slade, N. (1960). J Path., 13, 187.

Pereira, A. T. (1962). Ibid., 15, 252.

Shackman, R., and Messent. D. (1954). Brit. med. J., 2, 1009.

IIn a subsequent personal communication, Dr. Pereira states that: his described cases were of primary infections without predisposing lesions and without history of urethral instrumentation. Most of patients were out patients from different districts of Lisbon. 